\title{
Acesso público, autonomia financeira, agilidade editorial, novas indexações e homenagem aos pareceristas da RESR em 2019/2020
}

\author{
Public access, financial autonomy, agility in the editorial flow, new \\ indexing bases and a tribute to the reviewers of the RESR in 2019/2020 \\ Miguel Angelo Perondi ${ }^{1}$ (D) \\ ${ }^{1}$ Universidade Tecnológica Federal do Paraná (UTFPR), Curitiba (PR), Brasl. E-mail: perondi@utfpr.edu.br
}

Como citar: Perondi, M. A. (2020). Acesso público, autonomia financeira, agilidade editorial, novas indexações e homenagem aos pareceristas da RESR em 2019/2020. Revista de Economia e Sociologia Rural, 58(4), eED5804. https://doi.org/10.1590/1806-9479.2020.ED5804

\begin{abstract}
As responsabilidades do editor-chefe englobam a implementação da política editorial, a supervisão do processo editorial e as relações do periódico com os autores, pareceristas, leitores, indexadores, agências de apoio a pesquisa, a comunidade científica e o público geral. Em particular, a transparência e o controle de qualidade são aspectos essenciais do processo editorial sob a responsabilidade do editor-chefe. (Scientific Electronic Library Online, 2018, p. 02).
\end{abstract}

A Revista de Economia e Sociologia Rural (RESR) tem passado por profundas modificações, mas os desafios enfrentados em 2019 e 2020 são, no mínimo, inusitados. Ainda bem que se pode contar com uma preciosa equipe de trabalho, um "conselho editorial" prestativo, os carregadores de piano do "comitê científico" e o voluntariado dos pareceristas ad hoc que aperfeiçoaram os 90 artigos publicados nestes últimos dois anos, quase o dobro do mínimo requerido pela Plataforma SciELO Brasil (Scientific Electronic Library Online, 2017).

E para coroar o trabalho destes últimos dois anos, pretende-se aqui fazer uma análise dos bastidores da RESR aos seus colaboradores, procurando-se apresentar as questões-chave por trás das recentes conquistas. Para tanto, é importante se reportar às metas da RESR que foram pactuadas no $57^{\circ}$ Congresso da SOBER, em Ilhéus/BA, no ano 2019: (1) viabilizar o acesso on-line a 43 anos de acervo histórico da RESR em formato PDF editável (desde o volume 16 de 1978); (2) estabelecer a autonomia financeira; (3) agilizar o fluxo editorial; (4) melhorar a qualidade do fluxo editorial; e (5) incluir a revista em mais outra base de indexação. Assim, o texto, a seguir, analisa os avanços obtidos em cada uma dessas metas e, por fim, presta uma homenagem aos pareceristas da RESR que tornaram possível publicar os artigos dos anos de 2019 e 2020.

\section{Como a RESR obteve página própria e resgatou o seu acervo}

Em agosto de 2017, a RESR tinha um foco editorial voltado para a impressão gráfica e dispunha do acesso on-line exclusivamente por meio do portal SciELO, e este só abrangia os artigos publicados a partir de julho de 2002. Portanto, a RESR não tinha acesso on-line ao seu acervo em sua própria página. Ao mesmo tempo, a versão impressa não era disponibilizada aos sócios da Sociedade de Economia e Sociologia Rural (SOBER) nem aos colaboradores da RESR, mas somente aos seus poucos assinantes. Mesmo assim, a versão impressa era a maior identidade da RESR e era mantida pelo financiamento da SOBER e edital público do CNPq. 
Entretanto, a SOBER e o CNPq tiveram seus orçamentos reduzidos e, em 2020, acabaram por não mais financiar a RESR. Percebendo a gravidade crescente da escassez de recursos, 0 conselho editorial da RESR - na reunião do $56^{\circ}$ Congresso da SOBER, em Campinas/SP, em 2018 - autorizou o encerramento da versão impressa. Assim, o último número impresso foi v. 57, n. 1 da RESR, publicado em março de 2019. A partir de 2018, o desafio da RESR foi o de oportunizar o acesso público a todo o seu acervo em sua página, mesmo mantendo o acesso por meio do Portal SciELO.

Para tanto, contratou-se a plataforma PERIODIKOS, que possibilitou o acesso ao acervo da RESR em sua própria página. Inicialmente, cadastraram-se os 710 artigos (publicados entre 2002 e 2020) que estavam disponíveis na SciELO, tomando-se o cuidado de redirecionar o acesso ao PDF ao link oficial do DOI (Digital Object Identifier) para preservar a contagem de downloads dos artigos. Posteriormente, outros 755 artigos (publicados entre 1978 e 2002) foram digitalizados em formato pesquisável e incluídos no mesmo acervo da nova página da RESR (Revista de Economia e Sociologia Rural, 2020), a qual, portanto, disponibiliza 1.465 artigos, em formato PDF pesquisável e que foram publicados desde 1978.

\section{Os bastidores da autonomia financeira da RESR}

É importante salientar que a independência financeira da RESR em relação à SOBER não é decorrente somente da atual pandemia do Covid-19 que se abateu sobre o mundo em 2020 ou fruto da atual inoperância do Estado brasileiro, mas já vinha sendo gestada desde a Assembleia Geral do 54 Congresso da SOBER, em Maceió/AL, em 2016.

A primeira ação efetiva da busca da autonomia financeira foi aprovada na Assembleia Geral do $57^{\circ}$ Congresso da SOBER, em Ilhéus/BA, em 2019, quando se definiu que todo artigo aprovado a partir de $1^{\circ}$ de agosto de 2019 deveria contribuir com uma taxa de publicação no valor de $R \$ 600,00$. Nesse mesmo ano, também se estabeleceu um novo contrato com a Editora CUBO para terceirizar a etapa de publicação. Desta forma, publicaram-se o v. 57, n. 3 e o v. 57, n. 4 de 2019 e o v. 58, n. 1 de 2020, o que se estenderia ainda nos dois números seguintes. Entretanto, a escassez de financiamento externo obrigou a SOBER e a RESR a publicar uma carta conjunta estabelecendo que, a partir de 13 de abril de 2020, toda e qualquer publicação deveria colaborar com uma taxa de tramitação (no valor de $R \$ 100,00$ ) na submissão de um artigo para avaliação da Revista e, caso aprovado, colaborar com uma taxa de publicação.

Uma das mudanças decorrentes do processo de financiamento da publicação pelo próprio autor (não dependendo mais de subsídios para a publicação das edições) foi a instituição da publicação por fluxo contínuo a partir do v. 58, n. 1 de 2020. Neste caso, a periodicidade da RESR continuou sendo trimestral, no entanto, à medida que cada artigo passou a ser aprovado e editado, seguiu para ser publicado individualmente.

Para tanto, a partir de 2020, estabeleceu-se a paginação eletrônica (e-location), que permitiu desvincular a paginação de determinado artigo em relação aos demais do mesmo número. Para tanto, inseriu-se uma nota, na página inicial de todo artigo em fluxo contínuo, exemplificando como citar o artigo com base no modelo de referência bibliográfica APA (American Psychological Association).

Outra consequência imediata da cobrança pela taxa de tramitação, ou seja, a cobrança pela submissão, foi a redução de submissões de trabalhos ainda imaturos e frutos de disciplinas acadêmicas, o que melhorou muito a qualidade do fluxo editorial e a taxa de reprovação de submissões em decisão desk review pelo editor-chefe ou editor de área.

\section{Um olhar sobre os gargalos do fluxo editorial da RESR}

Para agilizar o fluxo editorial, uma ação implementada foi o de atualizar o sistema OJS para a versão 3.0 em junho de 2019, o que oportunizou uma visualização e gestão eletrônica da tramitação em uma versão muito mais "amigável". Ainda nesse ano, em busca de melhorar ainda mais a gestão editorial, iniciou-se a migração para o sistema operacional ScholarOne, para aprimorar os sistemas de controle e continuar reduzindo o tempo de tramitação das submissões. 
Outra inciativa para reduzir o tempo da tramitação foi, ao final de uma pré-avaliação positiva, o editor de área indicar mais do que três pareceristas vinculados ao tema, o que eleva a possibilidade de se encerrar a avaliação logo na primeira rodada.

Outra medida de controle foi estabelecer um teto máximo de tempo para cada etapa do fluxo editorial, que se encontra atualmente subdividido entre: (1) submissão (checagem normativa), (2) pré-avaliação (avaliação desk review), (3) tramitação (avaliação blind review) e (4) publicação (serviço terceirizado). Para tanto, espera-se não demorar mais do que 18 meses para publicar, seguindo as recomendações da SciELO (Scientific Electronic Library Online, 2017).

\section{Melhoria da qualidade editorial da RESR}

Desde janeiro de 2020, todos os atuais 20 componentes do comitê científico da RESR passaram a receber o status de editor de área e a assumir paulatinamente uma maior autonomia na administração do processo editorial da submissão sob seu encargo, tendo acesso a todos os dados. Com isso, obtêm-se agilidade e corresponsabilidade.

É importante registrar também que existe a experiência-piloto, desde janeiro de 2019, de se instituir a figura de um editor-adjunto das áreas de economia, administração e sociologia e desenvolvimento rural, o que torna mais rigorosa a avaliação desk review das submissões. Neste caso, ocorre uma divisão de trabalho entre o editor-chefe e os adjuntos, pois, enquanto o primeiro avalia a relevância temática, os adjuntos avaliam a relevância técnica da submissão. Essa dupla checagem de escopo reduz a demanda de pareceres ad hoc, priorizando-os para as submissões de elevada qualidade acadêmica e que corroboram o escopo da RESR.

Por fim, é importante salientar que a RESR propõe aos autores latinos que submetam o artigo de acordo com a sua língua nativa e traduzam depois de receber a carta de aceite, pois se prefere tramitar em português e traduzir a versão final para o inglês de forma profissional.

\section{A inclusão da RESR em novas bases de indexação}

A respeito de incluir a $R E S R$ em novas bases de indexação, considera-se uma meta que pode resultar em efetiva melhoria da indexação. Para tanto, já ocorreram as seguintes mudanças:

(a) No ano de 2019, indexou-se a RESR no DOAJ (Directory of Open Access Journal), cujo link se encontra na página principal da Revista.

(b) No primeiro trimestre de 2020, também se conseguiu indexar a RESR na base Researching Brazil, cujo link se encontra na página principal da Revista.

(c) Visando à indexação com LATINDEX e WEB OF SCIENCE, alguns componentes do comitê científico realizaram cursos promovidos pela ABEC (Associação Brasileira de Editores Científicos, 2020) em julho de 2020.

(d) É importante registrar que a participação em outras bases de indexação é estratégica para melhorar as avaliações por citação da RESR.

\section{Uma homenagem ao "parecerista desconhecido"}

A Revista de Economia e Sociologia Rural apresenta aqui sua homenagem aos 196 pareceristas que fizeram avaliação às cegas dos 90 artigos científicos publicados pela RESR entre 2019 e 2020. No Quadro 1, estão relacionados os nomes dos pareceristas em ordem alfabética e por país ou região. Assim, observa-se que: $6 \%$ são de residentes no exterior; 42\%, da região Sudeste; 31\%, do Sul; $11 \%$, do Nordeste; $9 \%$, do Centro-Oeste; e 1\%, do Norte. 
Quadro 1 - Pareceristas que contribuíram com a RESR entre 2019 e 2020.

\begin{tabular}{|c|c|c|c|}
\hline N. & PARECERISTA & INSTITUIÇÃO & PAÍS OU REGIÃO \\
\hline 1 & Herman Palau & UBA & Argentina \\
\hline 2 & Santiago Conti & CNICT & Argentina \\
\hline 3 & Maya Takagi & FAO & Chile \\
\hline 4 & Yormy Eliana Melo Poveda & UPB & Colômbia \\
\hline 5 & Isabel Dieguez Castrillon & UVIGO & Espanha \\
\hline 6 & Maria do Mar Pérez-Fra & USC & Espanha \\
\hline 7 & Fabio Mattos & UNL & EUA \\
\hline 8 & Peri Agostinho da Silva Junior & K-STATE & EUA \\
\hline 9 & Eric Pierre Sabourin & Cirad & França \\
\hline 10 & Gabriela Litre & CIRAD & França \\
\hline \multirow[t]{2}{*}{11} & Rui Manoel de Souza Fragoso & Uevora & Portugal \\
\hline & PARECERISTA SEDIADOS NO EXTERIOR & 11 & $6 \%$ \\
\hline 12 & Alcido Elenor Wander & Embrapa & Centro-Oeste \\
\hline 13 & Erlaine Binotto & UFGD & Centro-Oeste \\
\hline 14 & Gesmar Rosa Santos & IPEA & Centro-Oeste \\
\hline 15 & José Eustáquio Ribeiro Vieira Filho & IPEA & Centro-Oeste \\
\hline 16 & Jose Garcia Gasques & MAPA & Centro-Oeste \\
\hline 17 & José Humberto Valadares Xavier & Embrapa & Centro-Oeste \\
\hline 18 & Jose Roberto Rambo & UEMT & Centro-Oeste \\
\hline 19 & Leila Giandoni Ollaik & MPDG & Centro-Oeste \\
\hline 20 & Lindomar Pegorini Daniel & UNEMAT & Centro-Oeste \\
\hline 21 & Olivier François Vilpoux & UFMT & Centro-Oeste \\
\hline 22 & Otávio Valentim Balsadi & Embrapa & Centro-Oeste \\
\hline 23 & Regina Helena Rosa Sambuichi & IPEA & Centro-Oeste \\
\hline 24 & Richard Medeiros Araújo & CONAB & Centro-Oeste \\
\hline 25 & Rogerio Freitas & IPEA & Centro-Oeste \\
\hline 26 & Silvia Morales de Queiroz Caleman & UFMS & Centro-Oeste \\
\hline 27 & Sílvio Isoppo Porto & CONAB & Centro-Oeste \\
\hline 28 & Simone Maciel Cuiabano & UNB & Centro-Oeste \\
\hline \multirow[t]{2}{*}{29} & Thelma Lucchese Cheung & UFMS & Centro-Oeste \\
\hline & PARECERISTAS DA REGIÃO CENTRO-OESTE: & 18 & $9 \%$ \\
\hline 30 & Alexandre Rodrigues Loures & UFPB & Nordeste \\
\hline 31 & Amílcar Baiardi & UFBA & Nordeste \\
\hline 32 & Cimone Rozendo de Souza & UFRN & Nordeste \\
\hline 33 & Daniel Medeiros de Noronha Albuquerque & UFPI & Nordeste \\
\hline 34 & Eder Lucinda Pereira & CGU/UFT & Nordeste \\
\hline 35 & Eliane Pinheiro de Souza & URCA & Nordeste \\
\hline 36 & Emanoel Márcio Nunes & UERN & Nordeste \\
\hline 37 & Emerson Luís Lemos Marinho & UFC & Nordeste \\
\hline 38 & Francisco José Silva Tabosa & UFC & Nordeste \\
\hline 39 & Gisléia Benini Duarte & UFRPE & Nordeste \\
\hline 40 & Jair Andrade Araujo & UFC & Nordeste \\
\hline 41 & Janaina Ottonelli & UFBA & Nordeste \\
\hline 42 & Jean dos Santos Nascimento & UFCG & Nordeste \\
\hline 43 & Joacir Rufino de Aquino & UERN & Nordeste \\
\hline 44 & João Ricardo Ferreira de Lima & Embrapa & Nordeste \\
\hline
\end{tabular}


Quadro 1 - Continuação...

\begin{tabular}{|c|c|c|c|}
\hline N. & PARECERISTA & INSTITUIÇÃO & PAÍS OU REGIÃO \\
\hline 45 & Jorge Luiz Mariano Silva & UFPB & Nordeste \\
\hline 46 & Kilmer Coelho Campos & UFC & Nordeste \\
\hline 47 & Naisy Silva Soares & UESC & Nordeste \\
\hline 48 & Rodrigo Maciel Calvet & IFMA & Nordeste \\
\hline 49 & Tácito Augusto Farias & UFS & Nordeste \\
\hline 50 & Tales Wanderley Vital & UFRPE & Nordeste \\
\hline \multirow[t]{2}{*}{51} & William José Sabbag & UFRPE & Nordeste \\
\hline & PARECERISTAS DA REGIÃO NORDESTE: & 22 & $11 \%$ \\
\hline 52 & Alfredo Kingo Oyama Homma & Embrapa & Norte \\
\hline \multirow[t]{2}{*}{53} & Ima Célia Guimarães Vieira & Museu Goeldi & Norte \\
\hline & PARECERISTAS DA REGIÃO NORTE: & 2 & $1 \%$ \\
\hline 54 & Ademir de Lucas & USP & Sudeste \\
\hline 55 & Adriana Estela Sanjuan Montebello & UFSCAR & Sudeste \\
\hline 56 & Alan Figueiredo Arêdes & UFF & Sudeste \\
\hline 57 & Alexandre Bruno Pegorare & IBGE & Sudeste \\
\hline 58 & Alexandre Nunes Almeida & USP & Sudeste \\
\hline 59 & Aline Cristina da Cruz & UFSJ & Sudeste \\
\hline 60 & Ana Louise Carvalho Fiúza & UFV & Sudeste \\
\hline 61 & Antonio Carlos Lima Nogueira & FATEC & Sudeste \\
\hline 62 & Antonio Carlos Santos & UFLA & Sudeste \\
\hline 63 & Antonio Cesar Ortega & UFU & Sudeste \\
\hline 64 & Antônio Lázaro SantAna & UNESP & Sudeste \\
\hline 65 & Antônio Marcio Buainain & UNICAMP & Sudeste \\
\hline 66 & Arilson da Silva Favareto & UFABC & Sudeste \\
\hline 67 & Basilia Maria Baptista Aguirre & USP & Sudeste \\
\hline 68 & Bruna Branchi & PUC Campinas & Sudeste \\
\hline 69 & Bruno Benzaquen Perosa & UFU & Sudeste \\
\hline 70 & Bruno Pissinato & UNIMEP & Sudeste \\
\hline 71 & Candido Luiz de Lima Fernandes & IPEAD & Sudeste \\
\hline 72 & Carlos Eduardo de Freitas Vian & USP & Sudeste \\
\hline 73 & Carlos Enrique Guanzirolli & UFF & Sudeste \\
\hline 74 & Christiano Franca da Cunha & UNICAMP & Sudeste \\
\hline 75 & Clauber Eduardo Marchezan Scherer & UFMG & Sudeste \\
\hline 76 & Claudia Regina Rosal Carvalho & UFG & Sudeste \\
\hline 77 & Cristiane Feltre & PUC Campinas & Sudeste \\
\hline 78 & Daniel Henrique Dario Capitani & UNICAMP & Sudeste \\
\hline 79 & Daniela Carla Decaro Schettini & USP & Sudeste \\
\hline 80 & Delma Pessanha Neves & UFF & Sudeste \\
\hline 81 & Eduardo Delgado Assad & Embrapa & Sudeste \\
\hline 82 & Eduardo B. Monteiro de Castro Finamore & UFV & Sudeste \\
\hline 83 & Elli Fátima Napoleão de Lima & UFRural & Sudeste \\
\hline 84 & Érico Soriano & UFU & Sudeste \\
\hline 85 & Erly Teixeira & UFV & Sudeste \\
\hline 86 & Etiénne Groot & UNESP & Sudeste \\
\hline 87 & Evandro Camargo Teixeira & UFV & Sudeste \\
\hline 88 & Evandro Roberto Tagliaferro & UNIVBRASIL & Sudeste \\
\hline 89 & Fabiano Escher & UFRRJ & Sudeste \\
\hline
\end{tabular}


Quadro 1 - Continuação...

\begin{tabular}{|c|c|c|c|}
\hline N. & PARECERISTA & INSTITUIÇÃO & PAÍS OU REGIÃO \\
\hline 90 & Fernando Burgos Pimentel dos Santos & FGV & Sudeste \\
\hline 91 & Francisco Constantino Crocomo & FATEP & Sudeste \\
\hline 92 & Heliene Macedo de Araújo & UFSCAR & Sudeste \\
\hline 93 & Heloisa Lee Burnquisit & USP & Sudeste \\
\hline 94 & Humberto Francisco Silva Spolador & USP & Sudeste \\
\hline 95 & Isabela Nogueira & UFRJ & Sudeste \\
\hline 96 & Janice Rodrigues Placeres Borges & UFSCAR & Sudeste \\
\hline 97 & Jeronimo Alves dos Santos & UFSCAR & Sudeste \\
\hline 98 & José Alan Barbosa da Silva & Qualificar RH & Sudeste \\
\hline 99 & José Vicente Caixeta Filho & USP & Sudeste \\
\hline 100 & Juliana de Paula Filleti & FACAMP & Sudeste \\
\hline 101 & Juliano Costa Gonçalves & UFSCAR & Sudeste \\
\hline 102 & Júnia Cristina Péres Rodrigues da Conceição & USP & Sudeste \\
\hline 103 & Lechan Colares-Santos & UNOESTE & Sudeste \\
\hline 104 & Leonardo Chaves Borges Cardoso & UFV & Sudeste \\
\hline 105 & Leonilde Servolo de Medeiros & UFRRJ & Sudeste \\
\hline 106 & Lucas Siqueira de Castro & UFRural & Sudeste \\
\hline 107 & Luciana Florencio Almeida & ESPM & Sudeste \\
\hline 108 & Lucilio Rogerio Aparecido Alves & USP & Sudeste \\
\hline 109 & Lucimar Santiago de Abreu & UFSCAR & Sudeste \\
\hline 110 & Luiz Carlos Faria & UFSCAR & Sudeste \\
\hline 111 & Marcela Mello Brandão Vinholis & Embrapa & Sudeste \\
\hline 112 & Marcelo José Carrer & UFSCAR & Sudeste \\
\hline 113 & Maria José Teixeira Carneiro & UFRJ & Sudeste \\
\hline 114 & Maria Micheliana Costa Silva & UFV & Sudeste \\
\hline 115 & Maria Sylvia Macchione Saes & USP & Sudeste \\
\hline 116 & Marta Cristina Marjotta-Maistro & UFSCAR & Sudeste \\
\hline 117 & Mateus de Carvalho Reis Neves & UFV & Sudeste \\
\hline 118 & Nathalia Sbarai & UFVJ & Sudeste \\
\hline 119 & Omar Jorge Sabbag & UNESP & Sudeste \\
\hline 120 & Oscar Sarcinelli & UNICAMP & Sudeste \\
\hline 121 & Paulo Eduardo Moruzzi Marques & USP & Sudeste \\
\hline 122 & Raquel Castellucci Caruso Sachs & APTA & Sudeste \\
\hline 123 & Regina Aparecida Leite de Camargo & UNESP & Sudeste \\
\hline 124 & Rodrigo Lanna Franco da Silveira & UNICAMP & Sudeste \\
\hline 125 & Rodrigo Peixoto da Silva & PECEGE & Sudeste \\
\hline 126 & Rosa Livia Gonçalves Montenegro & UFJF & Sudeste \\
\hline 127 & Rosangela Aparecida de Medeiros Hespanhol & UNESP & Sudeste \\
\hline 128 & Samuel Alex Coelho Campos & UFF & Sudeste \\
\hline 129 & Sebastião Neto Ribeiro Guedes & UNESP & Sudeste \\
\hline 130 & Shigeo Shiki & UFU & Sudeste \\
\hline 131 & Silvio Y. M. Miyazaki & USP & Sudeste \\
\hline 132 & Valter Lúcio Oliveira & UFF & Sudeste \\
\hline 133 & Vanilde Ferreira Souza-Esquerdo & UNICAMP & Sudeste \\
\hline 134 & Vera Lucia Silveira Botta Ferrante & UNESP & Sudeste \\
\hline \multirow[t]{2}{*}{135} & Weslem Rodrigues Faria & UFJF & Sudeste \\
\hline & PARECERISTAS DA REGIÃO SUDESTE: & 81 & $42 \%$ \\
\hline
\end{tabular}


Quadro 1 - Continuação...

\begin{tabular}{|c|c|c|c|}
\hline N. & PARECERISTA & INSTITUIÇÃO & PAÍS OU REGIÃO \\
\hline 136 & Alexandre Florindo Alves & UEM & Sul \\
\hline 137 & Alexandre Giesel & UFFS & Sul \\
\hline 138 & Alisson Vicente Zarnott & UFSM & Sul \\
\hline 139 & Alysson Luiz Stege & UEPG & Sul \\
\hline 140 & Amanda M. de Souza Schuntzemberger & UFPR & Sul \\
\hline 141 & Ana Paula Myszczuk & UTFPR & Sul \\
\hline 142 & André Kuhn Raupp (2x) & IFC & Sul \\
\hline 143 & Anelise Graciele Rambo & UFRGS & Sul \\
\hline 144 & Angélica Massuquetti & UNISINOS & Sul \\
\hline 145 & Arlene Anelia Renk & UNOCHAPECÓ & Sul \\
\hline 146 & Augusto Mussi Alvim & PUC RS & Sul \\
\hline 147 & Bruno Anicet Bittencourt & UFRGS & Sul \\
\hline 148 & Carlos Alberto Gonçalves Jr & UNIOESTE & Sul \\
\hline 149 & Catia Grisa & UFRGS & Sul \\
\hline 150 & Chaiane Leal Agne & UERGS & Sul \\
\hline 151 & Cidonea Machado Deponti & UNISC & Sul \\
\hline 152 & Claudia Bueno Rocha Vidigal & UEM & Sul \\
\hline 153 & Cláudio Becker & UFPel & Sul \\
\hline 154 & Dimas de Oliveira Estevam & IAPAR & Sul \\
\hline 155 & Edison Luiz Leismann & UNIOESTE & Sul \\
\hline 156 & Ely José de Mattos & PUC RS & Sul \\
\hline 157 & Flávia Charão Marques & UFRGS & Sul \\
\hline 158 & Flávio Sacco dos Anjos & UFPel & Sul \\
\hline 159 & Gabrielito Rauter Menezes & UFPel & Sul \\
\hline 160 & Gilson Ditzel Santos & UTFPR & Sul \\
\hline 161 & Giovanna Pezarico & UTFPR & Sul \\
\hline 162 & Gustavo Inácio de Moraes & PUC & Sul \\
\hline 163 & Hieda Maria Pagliosa Corona & UTFPR & Sul \\
\hline 164 & José Donizetti de Lima & UTFPR & Sul \\
\hline 165 & José Marcos Froehlich & UFSM & Sul \\
\hline 166 & Josiane Carine Wedig & UTFPR & Sul \\
\hline 167 & Julyerme Matheus Tonin & UEM & Sul \\
\hline 168 & Junior Ruiz Garcia & UFPR & Sul \\
\hline 169 & Leonel Piovezana & UNOCHAPECO & Sul \\
\hline 170 & Luiz Ricardo Nakamura & UFSC & Sul \\
\hline 171 & Marcelo Antonio Conterato & UFRGS & Sul \\
\hline 172 & Marcelo Miele & Embrapa & Sul \\
\hline 173 & Marcia Dutra de Barcellos & UFRGS & Sul \\
\hline 174 & Marcio Gazolla & UTFPR & Sul \\
\hline 175 & Marco Antônio Verardi Fialho & UFSM & Sul \\
\hline 176 & Marcos Minoru Hasegawa & UFPR & Sul \\
\hline 177 & Maycon Noremberg Schubert & UFRGS & Sul \\
\hline 178 & Miguel Angelo Perondi & UTFPR & Sul \\
\hline 179 & Mirian Beatriz Schneider Braun & UNIOESTE & Sul \\
\hline 180 & Myriam Aldana Vargas Santin & FURB & Sul \\
\hline 181 & Norma Kiyota & IAPAR & Sul \\
\hline 182 & Oscar José Rover & UFSC & Sul \\
\hline
\end{tabular}


Quadro 1 - Continuação...

\begin{tabular}{cccc} 
N. & PARECERISTA & INSTITUIÇÃO & PAís OU REGIÃO \\
183 & Paulo Alexandre Nunes & UFFS & Sul \\
184 & Paulo André Niederle & UFRGS & Sul \\
185 & Paulo Dabdab Waquil & UFRGS & Sul \\
186 & Pedro Selvino Neumann & UFSM & Sul \\
187 & Rafael Gastal Porto & Embrapa & Sul \\
188 & Rene Eugenio Seifert Junior & UTFPR & Sul \\
189 & Rosane Marisa Spanevello & UFSM & Sul \\
190 & Rozane Marcia Triches & UFFS & Sul \\
191 & Sergio Schneider & UFRGS & Sul \\
192 & Sidemar Presotto Nunes & UTFPR & Sul \\
193 & Tatiana Walter & FURG & Sul \\
194 & Tatiele Lacerda da Silva & TJRS & Sul \\
195 & Valdemar João Wesz Junior & UNILA & Sul \\
196 & Veruschka Rocha Medeiros Andreolla & FURB & Sul \\
& PARECERISTAS DA REGIÃo SUL: & $\mathbf{6 0}$ & $\mathbf{3 1 \%}$ \\
\hline
\end{tabular}

Fonte: relatórios do OJS/SciELO de 2019 e 2020.

Assim, caro leitor, aproveite este espaço plural de debate interdisciplinar qualificado do mundo rural, ao mesmo tempo que se quer continuar contando com a crítica construtiva de seus leitores, autores e membros do conselho editorial e comitê científico, visando, a cada edição, aperfeiçoar o processo editorial.

\section{Nota de agradecimento:}

O editor agradece a leitura atenciosa e sugestões de melhoria deste texto aos colegas Paulo Eduardo Moruzzi Marques, Erlaine Binotto, Daniel Coronel, Joaquim Bento de Souza Ferreira Filho e Norma Kiyota.

\section{Referências Bibliográficas}

Associação Brasileira de Editores Científicos - ABEC BRASIL. (2020). Recuperado em 13 de julho de 2020, de https://www.abecbrasil.org.br

Revista de Economia e Sociologia Rural - RESR. (2020). Recuperado em 13 de julho de 2020, de https://www.revistasober.org/

Scientific Electronic Library Online - ScIELO. (2017). Critérios, política e procedimentos para a admissão e a permanência de periódicos na Coleção SciELO Brasil. Recuperado em 13 de julho de 2020, de http://www.scielo.br/avaliacao/Criterios_SciELO_Brasil_versao_revisada_atualizada_outubro_20171 206.pdf

Scientific Electronic Library Online - ScIELO. (2018). Guia de boas práticas para o fortalecimento da ética na publicação científica. Recuperado em 13 de julho de 2020, de https://wp.scielo.org/wpcontent/uploads/Guia-de-Boas-Praticas-para-o-Fortalecimento-da-Etica-na-PublicacaoCientifica.pdf

Informações sobre o autor: Editor Chefe da RESR (gestão 2017/19; 2019/21). Professor do Programa de Pós-Graduação em desenvolvimento Regional da UTFPR. 